\title{
Recent advances in using propofol by non-anesthesiologists Gang $\operatorname{Tan}^{1,2}$ and Michael G Irwin ${ }^{1 *}$
}

\author{
Addresses: ${ }^{1}$ Department of Anaesthesiology, University of Hong Kong, Room 424, Block K, Queen Mary Hospital, 102 Pokfulam Road, \\ Hong Kong; ${ }^{2}$ Department of Anaesthesiology, Peking Union Medical College Hospital, 100730, 1 Shuaifuyuan, Beijing, China \\ *Corresponding author: Michael G Irwin (mgirwin@hku.hk) \\ Fl000 Medicine Reports 2010, 2:79 (doi:10.3410/M2-79) \\ This is an open-access article distributed under the terms of the Creative Commons Attribution-Non Commercial License \\ (http://creativecommons.org/licenses/by-nc/3.0/legalcode), which permits unrestricted use, distribution, and reproduction in any medium, \\ provided the original work is properly cited. You may not use this work for commercial purposes. \\ The electronic version of this article is the complete one and can be found at: http://fl000.com/reports/m/2/79
}

\begin{abstract}
Evidence is accumulating that non-anesthesiologist-administered propofol (NAAP) sedation has a safety and efficacy profile comparable or superior to that provided by benzodiazepines with or without opioids. The guidelines currently available emphasize the importance of appropriate patient selection, staff training, monitoring, and low-dose sedation protocols for NAAP safety. In addition, capnograph monitoring and computer-assisted sedation systems may further improve patient safety during NAAP.
\end{abstract}

\section{Introduction and context}

Propofol (2,6 di-isopropyl phenol) is an ultra-shortacting anesthetic agent that provides sedative and amnestic effects at subanesthetic doses. It also has some analgesic effects [1]. Owing to its unique pharmacokinetic properties, its clinical applications have expanded since its introduction in the 1980s from the induction and maintenance of anesthesia to include monitored anesthesia care (MAC) and procedural sedation. Although in most product information it is stated that propofol should be administered only by anesthesiologists or persons with experience in intensive care, the use of non-anesthesiologist-administered propofol (NAAP) has dramatically increased, especially for gastrointestinal endoscopy [2].

Although such a potent anesthetic drug has well-known cardiorespiratory side effects, there are data from nonanesthesiologists suggesting that, using careful protocols, training, and monitoring, propofol sedation has a safety profile comparable to that of traditional sedation (benzodiazepine or opioids or their combination) in regard to the incidence of cardiopulmonary complications, airway interventions, or mortality [2-4]. NAAP resulted in faster induction of sedation and much shorter recovery times when compared with traditional sedation, and the rate of patient satisfaction was superior or equivalent $[3,4]$. Some endoscopists have even claimed that NAAP exhibits a safety profile comparable to that of general anesthesia or MAC and have questioned the costeffectiveness of anesthesiologist-administered sedation in gastrointestinal endoscopy $[2,3]$. In contrast, some anesthesiologists are worried that the use of propofol by unqualified persons may be responsible for increased complication rates [5]. One report concerning 153 deaths occurring after upper gastrointestinal endoscopy points out that high-risk patients (American Society of Anesthesiologists [ASA] III or higher) receiving intravenous sedation were not adequately monitored, and most of the deaths were attributed to the absence of an anesthesiologist during the procedure [6]. Currently, there are no studies directly comparing the safety and efficacy of NAAP with those of anesthesiologist-administered propofol sedation for gastrointestinal endoscopy or bronchoscopy.

Propofol has a narrow therapeutic window and a propensity to cause apnea, it also reduces airway muscle tone, produces cardiovascular depression, and does not have a reversal agent. In addition, sedation is a continuum of states [7], and a transition from minimal sedation to general anesthesia may occur inadvertently with a relatively small alteration in dose. Thus, it is relatively easy to induce oversedation and cardiopulmonary 
complications with propofol in endoscopic procedures that last a relatively long time, thereby requiring repeated doses or infusion of sedative agents, such as endoscopic retrograde cholangiopancreatography (ERCP), particularly in elderly high-risk patients. Although propofol sedation was associated with lower postinterventional hypoxemia and hepatic encephalopathy than benzodiazepines for older patients and patients with cardiorespiratory or liver disease $[8,9]$, there is a report showing that the safety of propofol sedation with careful monitoring was comparable to that of a midazolam/ pethidine combination for routine ERCP in elderly highrisk patients [10]. However, most guidelines or statements now available state that NAAP is appropriate for low- and average-risk patients undergoing elective endoscopic procedures [11-13] but that for high-risk patients NAAP is not recommended and an anesthetist should be available $[12,13]$.

There has been a general increase in minimally invasive surgery with a consequent demand for sedation services. In many settings, a shortage of staff means that anesthesiologists cannot provide services for all requested procedures outside the operating theater. Thus, NAAP appears to be a logical development for many procedures, particularly those less complicated ones such as screening gastrointestinal endoscopy. However, owing to the sociocultural, economic, and medico-legal differences that exist between countries, controversy over the legality of NAAP and how to use it safely and efficiently is still evident throughout the world.

\section{Recent advances}

Many articles demonstrating safety with NAAP, whether physician-administered or physician-directed nurseadministered propofol sedation, have been published. A recent multinational study of 646,080 gastrointestinal endoscopy procedures $(223,656$ from 28 published articles and 422,424 from 28 centers in 10 countries) revealed a very low overall risk of cardiopulmonary complications [2]. Of the 569,220 cases for which data were available, only $0.1 \%$ required transient bag-mask ventilation. The rates of bag-mask ventilation intervention were much lower for colonoscopy than upper endoscopy (0.01-0.10\%). Endotracheal intubation, permanent neurologic injury, and death resulted in 11, 0 , and 4 cases, respectively. The overall death rate was lower than the rate of reported deaths in cases sedated by endoscopists using opioids and benzodiazepines ( 1 per 160,000 as opposed to 11 per 100,000 ) [14]. The four patients who died all had severe coexisting diseases and received non-routine upper endoscopy procedures. Although the authors did not offer any information about the other seven intubated cases, the results suggest that NAAP can be performed safely for most gastrointestinal endoscopies but that those high-risk patients or procedures would benefit from specialist anesthesiologist care.

Aside from offering a comparable safety profile, NAAP provided a higher quality of sedation in terms of neuropsychometric recovery and patient tolerance [3,4,15-18]. Trummel and colleagues [15] used an expert videotape analysis method to evaluate the patient state during procedural sedation and found that propofol provided much better procedural conditions and greater patient satisfaction compared with a midazolam/opioid combination. Clark and colleagues [16] used the bispectral index (BIS) to direct propofol or midazolam sedation in flexible bronchoscopy and found that the induction time (the time required to achieve the targeted BIS value of 70-85 after drug injection) was similar in the two groups but that the recovery time (from the end of the procedure to recovery of BIS of greater than 90) was shorter in the propofol group $(5.4 \pm 4.7$ versus $11.7 \pm 10.2$ minutes $)$. The cognitive recovery evaluated by a continuous performance test at 15 minutes after the procedure also showed significant differences for all tested items in favor of the propofol group [16]. Horiuchi and colleagues [17] reported a clinical trial on nurseadministered low-dose propofol sedation for diagnostic esophagogastroduodenoscopy in 10,662 adults. Full recovery, including consciousness and psychomotor function, occurred in $99.9 \%$ of patients 60 minutes after the procedure. Most surprisingly, some of the patients even drove home after successful recovery with the permission of their physician [17]. Owing to its faster recovery profile, propofol sedation seems to improve patient satisfaction, and the efficiency of throughput also may be an economic advantage. It is important to stress that these data come from low-dose propofol sedation for relatively healthy individuals (without severe comorbidity) who underwent standard endoscopic procedures. The effect of propofol on patients with more comorbidity and the effect of higher doses of propofol (e.g., for longer-lasting interventional endoscopic procedures) remain unclear and need further research.

Since it is difficult to titrate single-agent propofol to moderate sedation and difficult to prevent patient movement or gagging during endoscopy, some endoscopists preferred 'balanced propofol sedation' (BPS). BPS combines propofol with a small dose of an opioid (e.g., fentanyl $50 \mu \mathrm{g}$ ) or a benzodiazepine (e.g., midazolam $1 \mathrm{mg}$ ) or both [19]. It is reported that BPS is safer because 
propofol requirements are lower, it is easier to titrate to moderate sedation, and it does not affect patient recovery time or satisfaction. However, the author of that report acknowledged that there is unquestionable synergism in the sedative effects of propofol with both opioids and benzodiazepines, thus the initial and repeated doses of propofol should be decreased accordingly and administered more carefully. Currently, there are no data available to compare the neuropsychomotor functional recovery after BPS to that of single-agent propofol sedation, and whether BPS increases postinterventional hypoxemia remains unclear. Randomized clinical trials that are well-designed and more detailed are needed to resolve these questions.

NAAP is usually done by giving repeated small-dose boluses in the same fashion that other agents for non-anesthesiologist-delivered sedation are given. The computer-assisted target-controlled infusion (TCI) system widely used by anesthesiologists in anesthesia and MAC is seldom mentioned by non-anesthesiologists [12]. The TCI system can automatically administer propofol through a computer-regulated infusion pump to achieve a particular target blood concentration. The dose and infusion rate are calculated by the computer according to previously validated three-compartment pharmacokinetic data adjusted to take into account patient demographic parameters (e.g., age, sex, height, and weight). The potential advantage of the TCI method is to simplify drug dosing and maintain a stable drug concentration and consequently a constant sedation depth. However, few data comparing TCI and traditional propofol administration methods for endoscopy exist. Recently, a computerassisted personalized sedation system (Sedasys; Ethicon Endo-Surgery, Inc., Cincinnati, OH, USA) in which propofol dosing is adjusted by a computer according to continuous physiologic monitoring has been developed. It has been approved by the ASA for clinical use and is indicated for minimal to moderate sedation in healthy adult patients (ASA physical status I or II) undergoing colonoscopy or esophagogastroduodenoscopy procedures. This device may be helpful for NAAP safety, but there are still very few data on its use [20].

As oxygen desaturation is actually a relatively late sign of respiratory obstruction or depression, capnograph monitoring has been introduced to promote NAAP safety. A recent study demonstrated that capnograph monitoring of respiratory activity during NAAP improves patient safety by reducing the frequency of hypoxemia, severe hypoxemia, and apnea [21]. Another report showed that, when the respiratory depression in adults receiving emergency department procedural propofol sedation was defined a priori as an $\mathrm{ETCO}_{2}$ (end-tidal carbon dioxide) level of $50 \mathrm{~mm} \mathrm{Hg}$ or greater, an absolute increase or decrease from baseline of $10 \%$ or greater, or a loss of waveform for 15 seconds or greater, the addition of capnography to standard monitoring identified all cases of hypoxia before onset, with the median time from capnographic evidence of respiratory depression to hypoxia being 60 seconds (range of 5-240 seconds) [22]. Capnography is worth recommending when it is difficult to visually assess respiration or during prolonged procedures such as ERCP and endoscopic ultrasound.

So far, there has been no technological advance that can replace professional expertise. Important elements of NAAP safety are a well-trained and appropriately qualified person or team, strict adherence to clear guidelines, and a cautious attitude. There are several different guidelines available for direct gastrointestinal endoscopistadministered propofol sedation $[7,12,13,23]$. The guidelines produced by the American Society for Gastrointestinal Endoscopy partially contradict those produced by the ASA for sedation by non-anesthesiologists [7], whereas the German [12] and Australian [13] guidelines were developed with the involvement of anesthesiologists. However, the following issues are common to all four guidelines: (a) the definition of the continuum of sedation; (b) a structured preprocedure patient evaluation, including informed consent for sedation; (c) standard monitoring during sedation with pulse oximetry, electrocardiogram, non-invasive blood pressure, respiration, and depth of sedation; and (d) special training of nonanesthesiologist physicians or nurses in pharmacology, complications recognition, and advanced life support, including the skills of airway management, defibrillation, and use of resuscitation drugs. The German and Australian guidelines agree that NAAP should be limited to patients of low and moderate risk, and the involvement of an anesthesiologist is recommended for high-risk patients. Both guidelines give a detailed definition of high-risk patients, but there is some discrepancy in the actual definitions. In the German guidelines, high-risk patients include high ASA classification (III or IV) and difficult endoscopic interventions or the presence of pathologicalanatomical features associated with a higher risk of airway obstruction during the intervention [12]. In the Australian guidelines, high-risk patients include (a) the elderly; (b) patients with severely limiting heart, cerebrovascular, lung, liver, or renal disease, morbid obesity, significant obstructive sleep apnea or known or suspected difficult endotracheal intubation, acute gastrointestinal bleeding with cardiovascular compromise or shock, severe anemia, the potential for aspiration of stomach contents (possibly necessitating endotracheal intubation), or previous adverse events due to sedation, analgesia, or anesthesia; and (c) ASA grade IV or V patients [13]. The guidelines 
formulated by the American Society for Gastrointestinal Endoscopy remain ambiguous with respect to the administration of sedation in ASA III or IV patients and state that the benefit of involving anesthesiologists in ASA IV (or higher) patients is unclear. This statement has been strongly rejected by some anesthesiologists and may well put high-risk patients at considerable risk [5]. A recent statement by the American Gastroenterology Association omits any recommendations on the administration of sedation in ASA III or IV patients and only mentions that the use of anesthesiologist-administered propofol for healthy individuals undergoing elective endoscopy without risk factors for sedation-related complications is very costly. The statement also advised that units initiating a propofol sedation program should do so under the direction of an anesthesiologist [3].

Although there are a few studies indicating that NAAP can be safely performed for bronchoscopy and some emergency department procedures $[4,16,22]$, much fewer outcome data are available for these procedures, and further research and audit are required before making any recommendation.

It has been reported that a tripartite group (the Australian and New Zealand College of Anesthetists, the Gastroenterological Society of Australia, and the Royal Australasian College of Surgeons, which conjointly established the guidelines for Australia) is in the process of establishing an endoscopist training program that involves the use of didactic lectures, small group discussions, anesthetic simulators, and observation sessions in units already using NAAP [24]. Such training programs are expected to legitimize NAAP and to help endoscopists use propofol safely and may be used as an example to other academic bodies.

\section{Implications for clinical practice}

Propofol use by non-anesthesiologists is likely to become more common because of increased demand by both patients and physicians and the increased number of procedures being performed outside the operating theater. If properly complied with, the various guidelines may contribute to the safe application of NAAP. Capnograph monitoring and computer-assisted personalized systems for propofol administration may help to prevent oversedation by non-anesthesiologists but these systems warrant further evaluation. The recovery time of propofol, especially the neuropsychometric recovery time with low-dose propofol titrated to moderate sedation, is much shorter than that of traditional sedation methods and drugs. Whether concurrent use of benzodiazepines/opioids is of benefit remains controversial and requires further evaluation.
Since propofol is very different from the more traditional sedative agents used by non-anesthesiologists, the units that are performing NAAP should be in close communication with anesthesiologists and make sure that anesthesia expertise is immediately available.

\section{Abbreviations}

ASA, American Society of Anesthesiologists; BIS, bispectral index; BPS, balanced propofol sedation; ERCP, endoscopic retrograde cholangiopancreatography; MAC, monitored anesthesia care; NAAP, non-anesthesiologistadministered propofol; TCI, target-controlled infusion.

\section{Competing interests}

The authors declare that they have no competing interests.

\section{References}

I. Bandschapp O, Filitz J, Ihmsen H, Berset A, Urwyler A, Koppert W, Ruppen W: Analgesic and antihyperalgesic properties of propofol in a human pain model. Anesthesiology 2010, I I 3:42 I-8.

2. Rex DK, Deenadayalu VP, Eid E, Imperiale TF, Walker JA, Sandhu K, Clarke AC, Hillman LC, Horiuchi A, Cohen LB, Heuss LT, Peter S, Beglinger C, Sinnott JA, Welton T, Rofail M, Subei I, Sleven R, Jordan P, Goff J, Gerstenberger PD, Munnings H, Tagle M, Sipe BW, Wehrmann T, Di Palma JA, Occhipinti KE, Barbi E, Riphaus A, Amann ST, Tohda G, McClellan T, Thueson C, Morse J, Meah N: Endoscopist-directed administration of propofol: a worldwide safety experience. Gastroenterology 2009, 137:1229-37.

FI000 Factor 10

Evaluated by Atul Kumar 22 Oct 2009

3. Vargo JJ, Cohen LB, Rex DK, Kwo PY: Position statement: nonanesthesiologist administration of propofol for GI endoscopy. Am J Gastroenterol 2009, 104:2886-92.

4. Bosslet GT, DeVito ML, Lahm T, Sheski FD, Mathur PN: Nurseadministered propofol sedation: feasibility and safety in bronchoscopy. Respiration 2010, 79:315-21.

5. Van der Linden P: Sedation in gastrointestinal endoscopy: an anesthesiologist's perspective. Digestion 2010, 82:102-5.

6. Thompson AM, Wright DJ, Murray W, Ritchie GL, Burton HD, Stonebridge PA: Analysis of 153 deaths after upper gastrointestinal endoscopy: room for improvement? Surg Endosc 2004, 18:22-5.

7. American Society of Anesthesiologists Task Force on Sedation and Analgesia by Non-Anesthesiologists: Practice guidelines for sedation and analgesia for non-anestheiologists. Anesthesiology 2002, 96:1004-17.

8. Qureshi WA, Zuckerman MJ, Adler DG, Davila RE, Egan JV, Ian Gan S, Lichtenstein DR, Rajan E, Shen B, Fanelli RD, Van Guilder T, Baron TH: ASGE guideline: modifications in endoscopic practice for the elderly. Gastrointest Endosc 2006, 63:566-9.

9. Riphaus A, Lechowicz I, Frenz MB, Wehrmann T: Profol sedation for upper GI endoscopy in patients with liver cirrhosis as alternative to avoid acute deterioration of minimal encephalopathy. A randomized controlled study. Scand J Gastroenterol 2009, 44: |244-5|.

10. Riphaus A, Stergiou N, Wehrmann T: Sedation with propofol for routine ERCP in high-risk octogenarians: a randomized, controlled study. Am J Gastroenterol 2005, I00:1957-63.

II. Cohen LB, Ladas SD, Vargo JJ, Paspatis GA, Bjorkman DJ, Van der Linden $P$, Axon AT, Axon AE, Bamias G, Despott E, Dinis-Ribeiro M, Fassoulaki A, Hofmann N, Karagiannis JA, Karamanolis D, Maurer W, O'Connor A, Paraskeva K, Schreiber F, Triantafyllou K, Viazis N, Vlachogiannakos J: Sedation in digestive endoscopy: the Athens 
international position statements. Aliment Pharmacol Ther 2010, 32:425-42.

12. Riphaus A, Wehrmann T, Weber B, Arnold J, Beilenhoff $U$, Bitter H, von Delius $S$, Domagk $D$, Ehlers AF, Faiss $S$, Hartmann $D$, Heinrichs W, Hermans ML, Hofmann C, In der Smitten S, Jung M, Kähler G, Kraus M, Martin J, Meining A, Radke J, Rösch T, Seifert H, Sieg A, Wigginghaus B, Kopp I: S3 Guideline: Sedation for gastrointestinal endoscopy 2008. Endoscopy 2009, 41:787-8I5.

13. Australian and New Zealand College of Anaesthetists; Gastroenterological Society of Australia; Royal Australasian College of Surgeons; Australasian College for Emergency Medicine; College of Intensive Care Medicine of Australia and New Zealand; Royal Australasian College of Dental Surgeons; Royal Australian and New Zealand College of Radiologists: Review PS9-Guidelines on sedation and/or analgesia for diagnostic and interventional medical, dental or surgical procedures - 2010. Melbourne, Australia; 2010. [http://www. anzca.edu.au/resources/professional-documents/ps9.html]

14. Sharma VK, Nguyen CC, Crowell MD, Lieberman DA, de Garmo P, Fleischer DE: A national study of cardiopulmonary unplanned events after GI endoscopy. Gastrointest Endosc 2007, 66:27-34.

15. Trummel JM, Surgenor SD, Cravero JP, Gordon SR, Blike GT: Comparison of differing sedation practice for upper endoscopic ultrasound using expert observational analysis of the procedural sedation. J Patient Saf 2009, 5:153-9.

16. Clark G, Licker M, Younossian AB, Soccal PM, Frey JG, Rochat T, Diaper J, Bridevaux PO, Tschopp JM: Titrated sedation with propofol or midazolam for flexible bronchoscopy: a randomised trial. Eur Respir J 2009, 34:1277-83.

17. Horiuchi A, Nakayama Y, Hidaka N, Ichise Y, Kajiyama M, Tanaka N: Lowdose propofol sedation for diagnostic esophagogastroduodenoscopy: results in 10,662 adults. Am J Gastroenterol 2009, 104:1650-5.
18. Kerker A, Hardt C, Schlief HE, Dumoulin FL: Combined sedation with midazolam/propofol for gastrointestinal endoscopy in elderly patients. BMC Gastroenterol 2010, 10: I I-5.

19. Rex DK: Endoscopist-directed propofol. Tech Gastrointest Endosc 2009, II:I77-80.

20. Pambianco DJ, Whitten C], Moerman A, Struys MM, Martin JF: An assessment of computer-assisted personalized sedation: a sedation delivery system to administer propofol for gastrointestinal endoscopy. Gastrointest Endosc 2008, 68:542-7.

21. Qadeer MA, Vargo JJ, Dumot JA, Lopez R, Trolli PA, Stevens T, Parsi M, Sanaka M, Zuccaro G: Capnographic monitoring of respiratory activity improves safety of sedation for endoscopic cholangiopancreatography and ultrasonography. Gastroenterology 2009, 136:1568-76.

FI000 Factor 6 Evaluated by Paul Kelly 19 Jun 2009

22. Deitch K, Miner J, Chudnofsky CR, Dominici P, Latta D: Does end tidal $\mathrm{CO} 2$ monitoring during emergency department procedural sedation and analgesia with propofol decrease the incidence of hypoxic events? A randomized, controlled trial. Ann Emerg Med 2010, 55:258-64.

23. Standards of Practice Committee of the American Society for Gastrointestinal Endoscopy, Lichtenstein DR, Jagannath S, Baron TH, Anderson MA, Banerjee S, Dominitz JA, Fanelli RD, Gan SI, Harrison ME, Ikenberry SO, Shen B, Stewart L, Khan K, Vargo Jj: Sedation and anesthesia in GI endoscopy. Gastrointest Endosc 2008, 68:8I5-26.

24. Thomson A, Andrew G, Jones DB: Optimal sedation for gastrointestinal endoscopy: review and recommendations. j Gastroenterol Hepatol 2010, 25:469-78. 